\author{
Marquette University \\ e-Publications@Marquette
}

Electrical and Computer Engineering Faculty

Research and Publications

Electrical and Computer Engineering,

Department of

\title{
Non-Local Model for the Spatial Distribution of Impact lonization Events in Avalanche Photodiodes
}

\author{
David A. Ramirez \\ University of New Mexico \\ Majeed M. Hayat \\ Marquette University, majeed.hayat@marquette.edu \\ Andrew S. Huntington \\ Voxtel, Inc. \\ George M. Williams \\ Voxtel, Inc.
}

Follow this and additional works at: https://epublications.marquette.edu/electric_fac

Part of the Computer Engineering Commons, and the Electrical and Computer Engineering Commons

\section{Recommended Citation}

Ramirez, David A.; Hayat, Majeed M.; Huntington, Andrew S.; and Williams, George M., "Non-Local Model for the Spatial Distribution of Impact Ionization Events in Avalanche Photodiodes" (2014). Electrical and Computer Engineering Faculty Research and Publications. 563.

https://epublications.marquette.edu/electric_fac/563 
Marquette University

e-Publications@Marquette

\section{Electrical and Computer Engineering Faculty Research and Publications/College of Engineering}

This paper is NOT THE PUBLISHED VERSION; but the author's final, peer-reviewed manuscript. The published version may be accessed by following the link in th citation below.

IEEE Photonics Technology Letters, Vol. 26, No. 1 (January 1, 2014): 25-28. DOI. This article is (C) Institute of Electrical and Electronic Engineers (IEEE) and permission has been granted for this version to appear in e-Publications@Marquette. Institute of Electrical and Electronic Engineers (IEEE) does not grant permission for this article to be further copied/distributed or hosted elsewhere without the express permission from Institute of Electrical and Electronic Engineers (IEEE).

\section{Non-Local Model for the Spatial Distribution of Impact lonization Events in Avalanche Photodiodes}

\section{David A. Ramirez}

Center for High Technology Materials, and the Department of Electrical and Computer Engineering, University of New Mexico, Albuquerque, NM

Majeed M. Hayat

Center for High Technology Materials, and the Department of Electrical and Computer Engineering, University of New Mexico, Albuquerque, NM

Andrew S. Huntington

Voxtel, Inc., Beaverton, OR

George M. Williams

Voxtel, Inc., Beaverton, OR 


\begin{abstract}
We report an extension of the analytical dead space multiplication theory that provides the means to theoretically determine the spatial distribution of electron and hole impact-ionization events in an arbitrarily specified heterojunction multiplication region. The model can be used to understand the role of the dead space in regularizing the locations of impact ionization. It can also be utilized to analyze, design, and optimize new generations of ultra-low noise, multistaged gain avalanche photodiodes based upon judiciously energizing and relaxing carriers to enhance electron impact ionization and suppress hole impact ionization.
\end{abstract}

\title{
SECTION I. Introduction
}

Avalanche photodiodes (APDs) are widely deployed in high-data-rate optical communications and laser radar systems that operate in the range of wavelengths from $950 \mathrm{~nm}$ to $1700 \mathrm{~nm}$. Among the APD structures, the separate absorption, charge and multiplication (SACM) InP-InGaAs APDs have been the preferred structure for two reasons. First, they have high sensitivity, which results from their internal carrier multiplication, namely the avalanche of impact ionizations that result from each photogenerated carrier. Second, they are highly cost effective compared to receivers that employ optical pre-amplification. However, due to the stochastic nature of the impact ionization process the multiplication gain comes at the expense of extra noise. This multiplication noise is characterized by a quantity termed the excess noise factor, which accounts for the gain uncertainty.

Various approaches have been explored to reduce the excess noise factor of APDs. They include the use of thin multiplication regions and impact-ionization engineered (I2E)multiplication regions. Both of these approaches exploit the dead-space effect to reduce the excess noise by making the spatial distribution of impact ionizations more deterministic [1], [2]. The dead space is the minimum distance a carrier must travel before it gains sufficient energy from the electric field to cause an impact ionization. Another approach is to suppress the impact ionization of holes (or alternatively, electrons), $\beta \rightarrow 0$ (or $\alpha \rightarrow 0$ ), to make $\alpha$ and $\beta$ as dissimilar as possible. According to McIntyre's local field theory [3], both the gain-bandwidth product and the excess noise of APDs improve when one of the ionization coefficients is much larger than the other [4], [5]. As such, there has been a growing interest in APD structures that suppress the impact ionization of holes (or electrons) by impactionization engineering of the multiplication region [6], [7]. In these structures the relaxation of one type of carrier (to prevent it from impact ionizing) is achieved by judiciously engineering the different layers of the heterojunction multiplication region and the electric field profile therein. A key factor in the successful design of this multi-layer multiplication regions is the ability to accurately determine the places at which electrons and holes trigger impact ionization events. While Monte-Carlo based simulation methods [6] exist to make such predictions, no theoretical method has yet been reported to the best of our knowledge.

In this letter we report an extension of the Dead Space Multiplication Theory (DSMT) [8] that enables the computation of the spatial distribution of the impact-ionization events within an arbitrarily specified heterojunction multiplication region without resorting to Monte-Carlo simulations. The newly developed recursive equations allow us to determine the number of electron and hole impact-ionization events individually within any sub-region of the multiplication region. Consequently, the developed model is a general model that is not limited to a particular structure of the multiplication region and, as such, it can be applied to a wide variety of multiplication-region designs. Preliminary results of the model were reported in [9]; however, no mathematical derivation was provided therein and the results were limited to showing the distribution of impact ionization events in two APD structures. In this letter we provide a rigorous mathematical derivation of the equations that describe the model. 


\section{SECTION II. Model}

Consider an arbitrary multiplication region extending from $x=0$ to $x=w$. We term an impact ionization event that is effected by a conduction-band electron an electron-ionization event; on the other hand, an impact ionization event that is effected by a valence-band hole is termed a hole-ionization event. Let $A$ be any subregion of the interval $[0, w]$. We will describe the equations that will enable us to solve the problem of computing the mean of the total number of electron-ionization events as well as the hole-ionization events occurring in the subregion $A$ after a single parent carrier (at a prescribed location) initiates the avalanche process. Note that if we can solve this problem for any subset $A$, then we can specialize it to the intervals $A_{1}=$ $[0, w / n), A_{2}=[w / n, 2 w / n), \ldots, A_{n}=[(n-1) w / n, w]$, and obtain the distribution of electron- and holeionization events throughout the multiplication region. (The partition parameter $n$ is selected to achieve a desired spatial distribution resolution.) To solve this problem, we define $Z_{e, A}(x)$ and $Z_{h, A}(x)$ as the total stochastic number of electron impact-ionization events and hole impact-ionization events, respectively, in the subregion $A$ when the avalanche process is triggered by a parent electron at location $x$. Similarly, we define $Y_{e, A}(x)$ and $Y_{h, A}(x)$ as the total stochastic number of electron impact-ionization events and hole impactionization events, respectively, in the subregion $A$ when the avalanche process is triggered by a parent hole at location $x$. Note that these quantities are different from the ones described in [8]. Specifically, here they represent the number of ionizations produced only by one species in a prescribed subregion while in [8] they represent the total number of offspring (in place of species-specific impact ionizations) in the entire multiplication region. Before proceeding with the formulation of recursive equations that enable us to solve for the ensemble averages of the quantities $Z_{e, A}(x)$ and $Z_{h, A}(x), Y_{e, A}(x)$ and $Y_{h, A}(x)$, let us formally introduce the probability density function of the distance from the birth location of a carrier to the location of its first impact ionization thereafter. Following the notation in [2], let $h_{e}(\xi \mid x)$ denote the pdf of the distance, $\xi$, to the first ionization measured from the electron's birth position at $x$. Similarly, $h_{h}(\xi \mid x)$ denotes the pdf of the distance traveled by a hole born at $\mathrm{x}$ before it first ionizes. In the DSMT, $h_{e}(\xi \mid x)$ and $h_{h}(\xi \mid x)$ are described by the shifted-exponential models

$h_{e}(\xi \mid x)= \begin{cases}\alpha(x+\xi) e^{-\int_{d_{e}(x)}^{\xi} \alpha(x+y) d y}, & \text { if } \xi \geq d_{e}(x) \\ 0, & \text { otherwise }\end{cases}$

and

$h_{h}(\xi \mid x)=\left\{\begin{array}{lc}\beta(x-\xi) e^{-\int_{d_{h}(x)}^{\xi}} \quad \beta(x-y) d y & \text { if } \xi \geq d_{h}(x)(2) \\ 0, & \text { otherwise, }\end{array}\right.$

where $d_{e}(x)$ and $d_{h}(x)$ are the electron and hole dead spaces, respectively. The exact formulas for calculating the dead space can be found elsewhere [2].

We now invoke a renewal argument, similar to that introduced in [8] to obtain recursive (integral) equations for the mean of the quantities $Z_{e, A}(x)$ and $Z_{h, A}(x), Y_{h, A}(x)$ and $Y_{h, A}(x)$, which we shall denote

as $z_{e, A}(x)$ and $z_{h, A}(x), y_{e, A}(x)$ and $y_{h, A}(x)$, respectively. Consider a parent electron at $x$ initiating the avalanche process and suppose that its first ionization occurs at some location $\xi>x$. If we assume (for the moment) that $\xi \notin A$, then the conditional mean of $Z_{e, A}(x)$ given that the first ionization has occurred at $\xi$ is simply $z_{e_{1}, A}(\xi)+z_{e_{2}, A}(\xi)+y_{e, A}(\xi)$, where $z_{e_{1}, A}(\xi)$ and $z_{e_{2}, A}(\xi)$ are the total average electron ionization events resulting from the two offspring electrons at $\xi$, while $y_{e, A}(\xi)$ is the total average electron ionization events resulting from the offspring hole at $\xi$. On the other hand, if the location $\xi$ of the first ionization is in $A$, 
then upon the first ionization we will already have had one electron ionization, and this addition has to be accounted for. In this case, the conditional mean of $z_{e, A}(x)$ is $1+z_{e_{1}, A}(\xi)+z_{e_{2}, A}(\xi)+y_{e}(\xi)$. Of course, there is a chance that the parent electron does not impact ionize at all (with probability $\int_{w}^{\infty} h_{e, A}(\xi \mid x) d \xi$, in which case $Z_{e, A}(x)$ be zero. By considering all of these scenarios while averaging over all possible locations $\xi$ of the location of the first impact ionization (by the parent electron) and upon further simplification we obtain the integral equation

$z_{e, A}(x)=\int_{A} h_{e}(\xi \mid x) d \xi+\int_{x}^{w}\left[2 z_{e, A}(\xi)+y_{e, A}(\xi)\right] h_{e}(\xi \mid x) d \xi,(3)$

where the first term is simply the probability that the first ionization occurs in the region $A$. We can repeat the same argument to analyze the ensemble averages of the quantities $Z_{h, A}(x), Y_{e, A}(x)$ and $Y_{h, A}(x)$; such analysis leads to three additional integral equations:

$y_{e, A}(x)=\int_{0}^{x}\left[2 y_{e, A}(\xi)+z_{e, A}(\xi)\right] h_{h}(\xi \mid x) d \xi$

$z_{h, A}(x)=\int_{x}^{w}\left[2 z_{e, A}(\xi)+y_{e, A}(\xi)\right] h_{e}(\xi \mid x) d \xi$

and

$y_{h, A}(x)=\int_{A} \quad h_{h}(\xi \mid x) d \xi+\int_{0}^{x}\left[2 y_{h, A}(\xi)+z_{h, A}(\xi)\right] h_{h}(\xi \mid x) d \xi$. (6)

The coupled recursive $\underline{(3)},(4), \underline{(5)}$ and $\underline{(6)}$ can be solved numerically using a simple iterative method.

\section{SECTION III. Results}

We have calculated the spatial distribution of electron- and hole-ionization events for two different cases of the multiplication region: (i) a hole-injection InP homojunction multiplication region, and (ii) an InAIAs/InAIGaAs electron-injection heterojunction multiplication region based on the multi-stage multiplication region reported in [10](See Fig. 1 in [10]). In our calculations we used the parameters of InP reported by Tan et al. [11], the parameters of $\operatorname{In}_{0.52} \mathrm{Al}_{0.48} \mathrm{As}$ reported by Saleh et al. [12], and the parameters of $\mathrm{In}_{0.53} \mathrm{Ga}_{0.47} \mathrm{As}$ reported by Pearsall [13] (Other impact ionization coefficients for $\operatorname{In}_{0.53} \mathrm{Ga}_{0.47}$ As that may be used are reported elsewhere [14]).

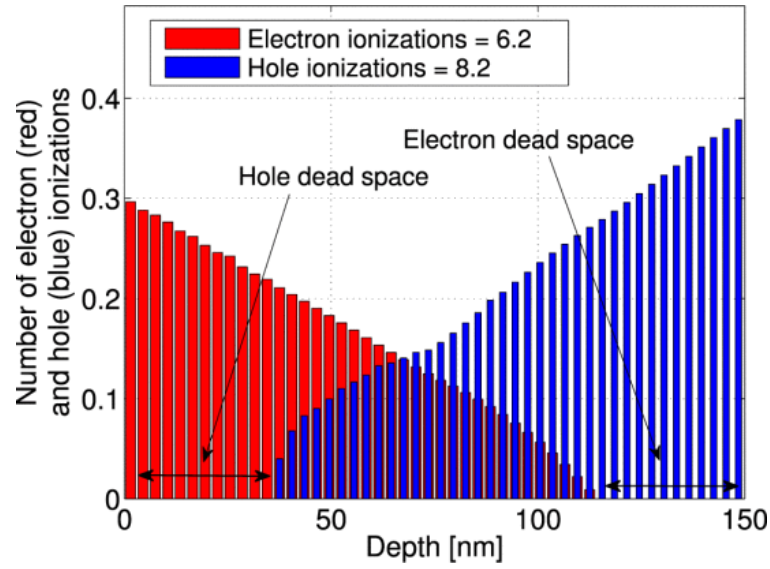

Fig. 1. Spatial distribution of electron-impact ionization (red) and hole-impact ionization (blue) events for a InP homojunction multiplication region of $150 \mathrm{~nm}$. The partition parameter, $n$, used in the calculations is 50 . 
Fig. 1 shows the calculated spatial distribution of electron-impact ionizations and hole-impact ionizations in an InP homojunction multiplication region of $150 \mathrm{~nm}$ under a constant electric field while using $n=50$. The mean multiplication gain is 15 . It is assumed that parent holes are injected at $x=0$. As expected, it can be seen from the figure that the number of hole-impact ionization events increases as the holes approach $x=150 \mathrm{~nm}$ while the number of electron impact ionizations events increases as the electrons approach $x=0$. This is consistent with the fact that holes and electrons multiply as they acquire sufficient kinetic energy from the electric field traveling in opposite directions. The figure also captures the effect of the dead space on the spatial distribution of the impact ionization events: the region in the beginning of the multiplication region, from $x=0$ to $x \approx$ $37 \mathrm{~nm}$, exhibits no hole impact ionizations due to the dead space of the holes in this region. Similarly, in the region at the end of the multiplication region, from $\mathrm{x} \approx 112 \mathrm{~nm}$ to $x=150 \mathrm{~nm}$, no electron impact ionizations are observed, as expected due to the electrons' dead space in this region.

We have also calculated the spatial distribution of the electron- and hole-impact ionization events considering a single-carrier multiplication (SCM) APD with an InAIAs/InAIGaAs multiplication region. SCM-APDs were developed by Voxtel Inc. to obtain quasi-deterministic multiplication gains by suppressing hole impact ionization events [7], [10]. The multiplication region of an SCM-APD consists of a cascaded multiplier architecture, which combines various design techniques to suppress hole ionizations and enhance electron ionizations [7], [10]. Fig. 2 shows the electric field profile across the multiplication region. The multiplication region has 5 multiplication cells, each of which consists of an avalanche layer, a hole relaxation layer, and an electron heating layer. The inset of Fig. 2 shows electric-field profile of the first two multiplications cells and the corresponding layers inside the cells. We assume that parent electrons are injected at $x=0$. To incorporate carrier relaxation due to phonon scattering we replace the nonlocalized ionization coefficients $\alpha(x)$ and $\beta(x)$ (in (1) and (2)) by the so-called "scattering-aware" ionization coefficients $\bar{\alpha}(\xi \mid x)$ and $\bar{\beta}(\xi \mid x)$, respectively [7]. In addition, we also need to replace $d_{e}(x)$ and $d_{h}(x)$ by their scattering-aware counterparts $\overline{d_{e}}(x)$ and $d_{h}(x)$, respectively. To calculate $\bar{\alpha}(\xi \mid x), \bar{\beta}(\xi \mid x), \overline{d_{e}}(x)$, and $\overline{d_{h}}(x)$ we assume that a carrier loses its energy and ceases to be able to impact-ionize if it travels a certain nominal distance during which its impact ionization coefficient ( $\alpha$ for electrons and $\beta$ for holes) is below a certain threshold $\left(30 \mathrm{~nm}\right.$ below $10^{3} \mathrm{~cm}^{-1}$ ). Here, we assume that the energy accumulated by a carrier is reset to zero once the carrier travels the nominal distance under a field that falls below a certain threshold. For completeness, we briefly describe the scattering-aware ionization coefficients, $\bar{\alpha}(\xi \mid x)$ and $\bar{\beta}(\xi \mid x)$ drawing freely from [7]. The quantity $\bar{\alpha}(\xi \mid x)$ is the ionization coefficient at $\xi$ for an electron that has zero energy at location $x$, with $x<\xi$, and $\bar{\beta}(\xi \mid x)$ is the ionization coefficient at $\xi$ for a hole that has zero energy at location $x$, with $x>\xi$. Note that these coefficients are dependent on the entire field profile from $x$ to $\xi$, not simply on the field at $\xi$. The quantities $\overline{d_{e}}(x)$ and $\overline{d_{h}}(x)$ denote the dead spaces that an electron and hole, respectively, created at location $x$ in the multiplication region must travel before they can accumulate their ionization threshold energy in the material. For an electron starting at location $\mathrm{x}$ with zero energy reserve, we set $\bar{\alpha}(\xi \mid x)=0$ if $\xi<x+\overline{d_{e}}(x)$. However, if $\xi \geq x+\overline{d_{e}}(x)$, then we find the last point, $s$, at which the energy of the electron was reset to zero, and then check whether the electron has traveled the dead

space $\overline{d_{e}}(s)$ beyond the point s. If indeed it has traveled $\overline{d_{e}}(s)$ beyond s, we set $\bar{\alpha}(\xi \mid x)=\alpha(\xi)$, where $\alpha(\xi)$ is the usual nonlocalized ionization coefficient (also termed enabled ionization coefficient). On the other hand, if the electron has not traveled a distance $\overline{d_{e}}(s)$ beyond $s$, we set $\bar{\alpha}(\xi \mid x)=\alpha(\xi)$. The scattering-aware ionization coefficient for the holes is calculated similarly. Once the scattering-aware ionization coefficients are calculated, they are substituted for the nonlocalized ionization coefficients in (1) and (2). More details about the scatteringaware DSMT are provided by Williams et al. [7]. In [10], the scattering-aware DSMT was used to calculate the gain and excess noise factor of a 10-stage InAIAs/InGaAs single carrier multiplication APD reported therein. The results of the model are consistent with Voxtel's Monte-Carlo simulations and experimental results [10]. 


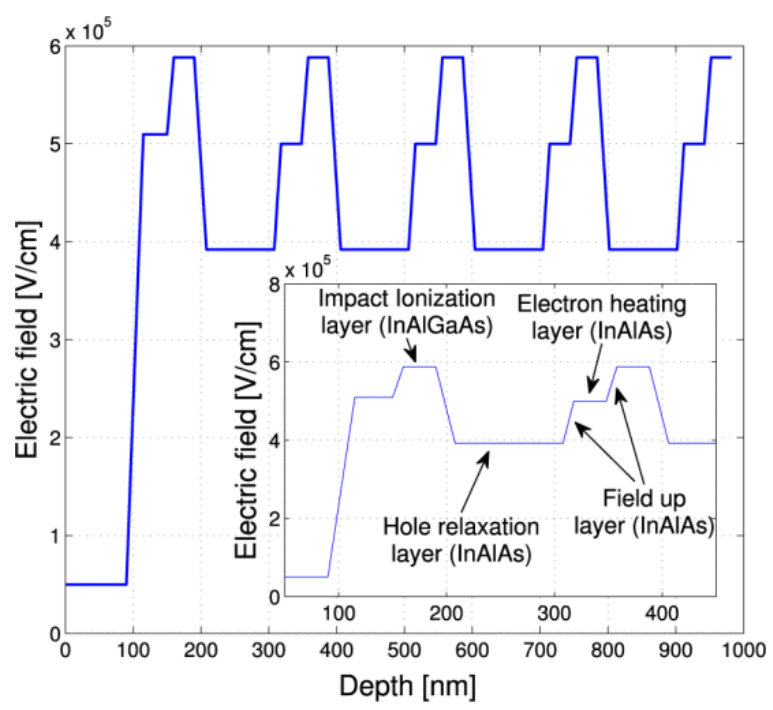

Fig. 2. Electric-field profile across the InAIAs/InAIGaAs SCM APD multiplication region. The inset shows electricfield profile of the first two multiplications cells and the corresponding layers inside the cells. Details about the design of the multiplication cells can be found elsewhere [7], [10].

Fig. 3 shows the distribution of the electron- and hole-ionization events in an SCM-APD with a multiplication region of $1 \mu \mathrm{m}$. The electric field across this multiplication region is shown in Fig. 2. It can be seen that most of the impact ionization events occur in the lower band-gap and high ionization rate layers (InAIGaAs), which are the layers where the electric field is at a maximum. The figure also shows the large difference between the number of electron-impact ionizations compared to that of hole-impact ionizations. This disparity is a result of two factors: (1) the hole-relaxation layers, which prevent the holes form acquiring sufficient kinetic energy to impact ionize and subsequently reducing excess noise, and (2) the electrons are pre-heated prior injection into the InAIGaAs layer.

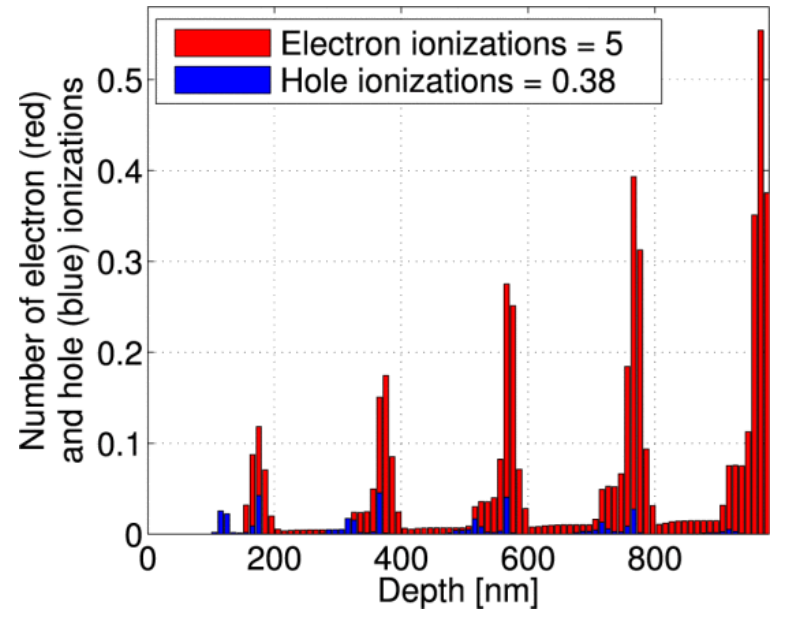

Fig. 3. Spatial distribution of electron-impact ionization (red) and hole-impact ionization (blue) events for an SCM-APD with a multiplication region of $1 \mu \mathrm{m}$. The partition parameter, $n$, used in the calculations is 100 .

\section{SECTION IV. Discussion}

The reported model can be very useful for understanding and designing APDs in which the locations where impact ionization events take place are controlled to improve device performance. Examples of this type of devices are heterostructure APDs designed to achieve ultra-low excess noise performance by suppressing the 
impact ionizations due to holes [1], [7], [10]. In addition, the model is capable of handling nonuniform electric fields and position-dependent ionization coefficients. However, as an analytical approximation of the impact ionization process, this model is based on assumptions that are accurate under certain conditions. Specifically, the model assumes that after a carrier has traveled a minimum distance given by the dead-space, it will impact ionize according to a probability density function that depends only on the electric field and material-specific ionization-model parameters along the path to ionization. These assumptions are accurate for electric-field profiles that change slowly across the multiplication region. In particular, our model allows the ionization coefficients to change instantaneously as carriers cross heterojunctions where the electric field and material change abruptly. However, when the electric field changes abruptly the hot carriers will not be in equilibrium with the electric field instantaneously. Therefore, while our analytical model provides a good and computationally efficient approximation to the problem of avalanche multiplication in complex heterojunctions, a more accurate approach would require the use, for example, of full-band Monte-Carlo simulations [15]. It is important to note that the DSMT has been experimentally validated in bandgap-engineered heterojunction multiplication regions (e.g., see [2]) and the scattering-aware DSMT has also been experimentally validated [7].

\section{SECTION V. Conclusion}

We have developed a theoretical recursive model to separately determine the spatial distribution of electronionization events and hole-ionization events as the avalanche process is triggered by either a parent hole or electron at an arbitrary location inside multiplication region. The new model is a useful theoretical tool for understanding, designing and optimizing new generations of APDs designed to achieve ultra-low noise characteristics by enhancing impact ionizations of electrons (holes) while suppressing impact the ionizations of holes (electrons).

\section{Refernces}

1. S. Wang, "Ultra-low noise avalanche photodiodes with a 'centered-well' multiplication region", IEEE J. Quantum Electron., vol. 39, pp. 375-378, Feb. 2003.

2. M. M. Hayat, O.-H. Kwon, S. Wang, J. C. Campbell, B. E. A. Saleh, M. C. Teich, "Boundary effects on multiplication noise in thin heterostructure avalanche photodiodes: Theory and experiment $\backslash \$[\mathrm{rm}$ Al\}_\{0.6\}\{rm Ga\}_\{0.4\}\{rm As $\} /\{\mathrm{rm} \mathrm{GaAs}\}] \backslash \$$ ", IEEE Trans. Electron Devices, vol. 49, pp. 2114-2123, Dec. 2002.

3. R. J. McIntyre, "Multiplication noise in uniform avalanche photodiodes", IEEE Trans. Electron Devices, vol. 13, pp. 164-168, Jan. 1966.

4. J. P. R. David, C. H. Tan, "Material considerations for avalanche photodiodes", IEEE J. Sel. Topics Quantum Electron., vol. 14, no. 4, pp. 998-1009, Jul./Aug. 2008.

5. J. C. Campbell, "Recent advances in telecommunications avalanche photodiodes", J. Lightw. Technol., vol. 25, no. 1, pp. 109-121, Jan. 2007.

6. W. Sun, X. Zheng, Z. Lu, J. C. Campbell, "Monte Carlo simulation of InAIAs/InAIGaAs tandem avalanche photodiodes", IEEE J. Quantum Electron., vol. 48, pp. 528-532, Apr. 2012.

7. G. M. Williams, M. Compton, D. A. Ramirez, M. M. Hayat, A. S. Huntington, "Multi-gain-stage InGaAs avalanche photodiode with enhanced gain and reduced excess noise", IEEE J. Electron Devices Soc., vol. 1, no. 2, pp. 54-65, Feb. 2013.

8. M. M. Hayat, B. E. A. Saleh, M. C. Teich, "Effect of dead space on gain and noise of double-carrier multiplication avalanche photodiodes", IEEE Trans. Electron Devices, vol. 39, pp. 546-552, Mar. 1992.

9. D. A. Ramirez, M. M. Hayat, A. S. Huntington, G. M. Williams, "Theory for spatial distribution of impactionization events in avalanche photodiodes", Proc. IEEE IPC, pp. 100-101, 2012-Oct.

10. G. M. Williams, D. A. Ramirez, M. M. Hayat, A. S. Huntington, "Time resolved gain and excess noise properties of InGaAs/InAlAs avalanche photodiodes with cascaded discrete gain layer multiplication regions", J. Appl. Phys., vol. 9, pp. 093705-1-093705-11, Mar. 2013. 
11. L. J. J. Tan, J. S. Ng, C. H. Tan, J. P. R. David, "Avalanche noise characteristics in submicron InP diodes", IEEE J. Quantum Electron., vol. 44, pp. 378-382, Apr. 2008.

12. M. A. Saleh, "Impact-ionization and noise characteristics of thin III-V avalanche photodiodes", IEEE Trans. Electron Devices, vol. 48, pp. 2722-2731, Dec. 2001.

13. T. P. Pearsall, " Impact ionization rates for electrons and holes in $\backslash \$\{r m ~ G a\} \_\{0.47\}\{r m$ In $\}$ \{0.53\}\{rm As $\} \backslash \$$ ", Appl. Phys. Lett., vol. 36, no. 3, pp. 218-220, Feb. 1980.

14. F. Osaka, T. Mikawa, T. Kaneda, "Impact ionization coefficients of electrons and holes in (100)-oriented $\backslash \$\{r m \text { Ga }\}_{-}\{1-x\}\{\mathrm{rm} \mathrm{In}\}\left\{\{x\}\{r m \text { As }\}_{-}\{y\}\{r m \text { P }\}_{-}\{1-y\} \backslash \$\right.$ ", IEEE J. Quantum Electron., vol. 21, pp. 1326-1338, Aug. 1985.

15. D. S. Ong, K. F. Li, S. A. Plimmer, G. J. Rees, J. P. R. David, P. N. Robson, " Full band Monte Carlo modeling of impact ionization avalanche multiplication and noise in submicron $\mathrm{GaAs} \backslash \$\{\mathrm{rm} p\}^{\wedge}\{+\} \mathrm{hbox}\{-\}\{\mathrm{rm}$ i $\} \mathrm{hbox}\{-$ \}$\{r m n\}^{\wedge}\{+\} \backslash \$$ diodes ", J. Appl. Phys., vol. 87, no. 11, pp. 7885-7891, 2000. 